\title{
Towards Unsupervised Weed Scouting for Agricultural Robotics
}

\author{
David Hall ${ }^{1}$
}

\author{
Feras Dayoub ${ }^{2}$
}

\author{
Jason Kulk ${ }^{1}$ \\ Chris $\mathrm{McCool}^{1}$
}

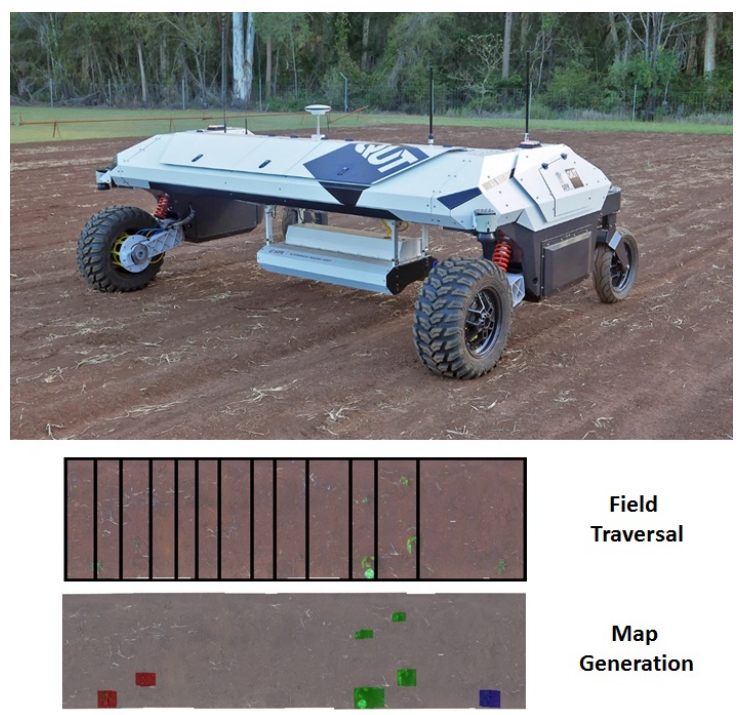

Fig. 1: Simplified demonstration of weed scouting where the AgBotII robotic platform traverses the field and marks out weed locations. Each coloured box represents a different weed group. Field traversal image has undergone some image enhancement for visualization purposes. Best viewed in colour.

advance in order to generate weed distribution maps. Weeds are clustered into visually similar groups which can then be identified by farmers without requiring manual inspection of each plant or a prior knowledge about the field. We adapt current clustering algorithms to improve them for our task and demonstrate the first results of our clustering approach to weed scouting utilizing data collected from the AgBotII agricultural robotics platform (shown in Fig. 1) showing its applicability to real world conditions.

The contributions supplied in this paper are as follows. We introduce a set of deep convolutional neural network (DCNN) bottleneck features trained on an unrelated plant dataset to be used within our unsupervised system for weed scouting, showing that these can improve upon results using normal DCNN features. We introduce an image-locking system for hierarchical-type clustering algorithms which takes advantage of multiple tracked plant observations to improve clustering accuracy. Finally, we introduce a new applicationoriented evaluation metric.

\section{LiterATURE REVIEW}

Commonly, weed scouting has been done through a manual inspection of the field. However, and as shown in [4],

\footnotetext{
${ }^{1}$ The authors are with the School of Electrical Engineering and Computer Science, Queensland University of Technology (QUT), Brisbane, Australia. email: $\{$ d20.hall, jason.kulk, c.mccool $\} @$ qut.edu.au

2 The author is with the ARC Centre of Excellence for Robotic Vision, Queensland University of Technology (QUT), Brisbane, Australia. http://www.roboticvision.org/email: feras.dayoub@qut.edu.au
} 
autonomous weed scouting can reduce costs by $20 \%$ over manual weed inspection.

In previous attempts at automated weed scouting and destruction, there have been two main approaches, either through treating all weeds as being the same [5], or through species classification [6]. The first approach, while effective for choosing herbicide dosages and examining where the highest infestations occur, is not particularly useful for integrated weed management strategies where each weed species must be treated in an appropriate manner. The second approach uses supervised classification, which can achieve high accuracy, but requires a priori knowledge of the weed species present in the field so that it can be trained to recognise them. These issues restrict the widespread deployment of such approaches.

Another approach which can address this problem is weed scouting based on unsupervised methods such as clustering. Clustering is the procedure of forming meaningful groups from samples [7]. In our case, the samples are images of weed plants in the field and the groups are the species.

Clustering can be done with or without knowing the number of clusters $(K)$. Clustering methods which need a known $K$ like k-means [8] are unrealistic for a scouting scenario where there can be no certainty about how many groups will be required. Therefore in this work, we focus on clustering algorithms which estimate $K$ as a part of the clustering process.

Of the techniques that do not require a known number of clusters, one of the most popular is hierarchical clustering which aims to iteratively merge or split clusters based on a distance metric between clusters in order to form cluster relationship trees [9]. This allows for a more adaptable clustering system, with the number of clusters being calculated after the full trees have been computed, often through some stopping criterion being reached [10]. In [11], however, it was pointed out that hierarchical clustering is a static system where points once assigned to one cluster cannot be allocated to a new cluster, allowing for errors to be propagated through the system.

In order to overcome such problems, variations to hierarchical clustering have been developed. Competitive agglomerative clustering, demonstrated in [12] and [11], does not merge clusters in the same sense as is done in hierarchical clustering. Instead, clusters competes over the samples available and are slowly removed as their samples are taken by larger clusters. This is similar to what is done within infinite Gaussian mixture models (IGMMs) and Dirichlet process mixture models (DPMMs) which iteratively go through a process of reassigning points to existing clusters and removes clusters through this process [13].

Another approach which is able to perform clustering without knowing the number of clusters is affinity propagation [14], [15]. This powerful technique generates exemplars by iteratively updating how likely the each sample can serve as an exemplar and how likely each sample could belong to each point as an exemplar. Through this process, eventually a consensus occurs and clusters are assigned to all samples.
An approach which is more akin to traditional hierarchical clustering but which still takes measures to allow for reassigning data points is the hierarchical approach utilized in diarization [16]. Diarization systems are used heavily in signal processing and attempt to answer the question "who spoke when?" [17]. They comprise of two elements, the segmentation of audio data into speaker and non-speaker segments and clustering speaker segments into specific speaker groups with each group corresponding to one individual. The clustering used in diarization differs from hierarchical clustering in that after merging two clusters, data is resegmented and the clusters retrained .

Another important aspect of weed scouting based on clustering is image features. In recent years the development of features learnt using deep convolutional neural networks [18] have proved beneficial in many recognition tasks. Deep neural network architectures such as those demonstrated in [19] and [20] can be fine-tuned as described in [18] in order to obtain features more suited to the precise recognition task to be performed.

As shown above, current automated weed scouting methods are based on supervised learning methods which need a priori knowledge about the weeds that exist in the field. This defies the purpose of the scouting process (i.e finding what weed plants exist in the field). This shortcoming makes unsupervised clustering methods an attractive solution and more suitable approach for the task.

\section{Methodology}

The algorithm used in our weed scouting system for agricultural robotics consists of four main stages. These are plant detection and segmentation, feature extraction, species clustering, and field mapping. The following sections explain these stages in more detail.

\section{A. Plant Detection and Segmentation}

In our work, detection was performed using only colour imagery without any extra multi-spectral imaging. Inspired by [21], our segmentation method utilizes multiple colour spaces for weed detection. The colour spaces utilized are summarized below.

HSV is a cylindrical colour space consisting of hue $(\mathrm{H})$, saturation (S) and value (V), or brightness.

Luv is a perceptually uniform colour space where lightness is captured by the component $\mathrm{L}$.

$\mathrm{Lab}$ is an opponent colour space where $\mathrm{a}$ and $\mathrm{b}$ are the colour-opponent dimensions.

Our plant detector uses a multivariate Gaussian trained with the following feature vector $[\mathrm{H}, \mathrm{S}, \mathrm{u}, \mathrm{v}, \mathrm{a}, \mathrm{b}]$. Model parameters and the threshold probability for the Gaussian are learnt on a training set of manually annotated images which are not related to the dataset used in our clustering experiments. The full detection pipeline is shown in Fig 2. When applied to a new image, the Gaussian provides a per-pixel log-likelihood map, or segmentation map, similar to that in Figure 2 (b). As this is often noisy, to convert the segmentation map into weed regions we first remove noise from the image and then 


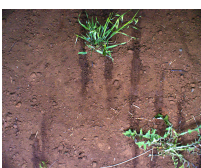

(a)

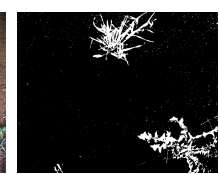

(b)

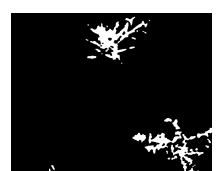

(c)

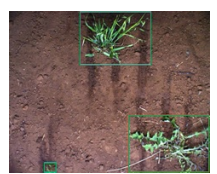

(d)
Fig. 2: From left to right (a) the original image, (b) the result of per-pixel segmentation after thresholding, (c) the filtered image after erosions and dilations (final mask), and (d) the detection regions, indicated by the bounding boxes.

search for connected regions. Contiguous regions are found from this binary image by finding the contours [22] and those which are close are merged to form a single weed segmentation mask which can be utilized later on in our clustering pipeline for feature extraction.

\section{B. Feature Extraction}

Effective plant species clustering is highly dependant on the feature representation given for each plant. In our experiments we use and evaluate two main categories of feature, these being hand-crafted features (HCFs) typically used in plant classification for agricultural robotics and learnt deep convolutional neural network (DCNN) features.

We utilize the same HCFs as are used within [23] and [24], consisting of the following shape features: plant perimeter, plant area, length of plant mask skeleton, compactness, convexity, length of plant mask skeleton/plant perimeter; and the following statistical reflectance features: minimum, maximum, range, mean, median, standard deviation, kurtosis, and skewness of the plant pixel intensities. Further detail of how each of these features are extracted can be found within [24]. As in [23], we analyse the use of two sets of HCFs, one containing all of the shape and reflectance features described, and a scale-robust subset which discludes the features which are affected by image scale (perimeter, area, and length of skeleton). These two feature sets will be referred to as hcf and hcf-scale-robust within this paper.

We also used deep DCNN features for our clustering experiments. Namely, the powerful Inception architecture [20] known as GoogLeNet which is a deep network with 22 layers. This is first pre-trained on ImageNet and fine-tuned on a training subset of the leaf images from the PlantCLEF dataset [25].

While learnt features are known to be highly descriptive, they come a cost of high dimensionality. It is known that a high dimension feature representation can cause a clustering system to suffer due to the "curse of dimensionality". In order to alleviate this issue, we follow the procedure shown in [26] to generate so called bottleneck features which are both discriminative and low dimensional. This network is fine-tuned on the same training images from PlantCLEF as the original architecture. We shall refer to these as bottleneck features throughout this paper. $\overline{\text { Algorithm } 1 \text { Our diarization clustering algorithm consists of }}$ an over segmented cluster initialization (line 2) followed by a repeated loop of cluster resegmentation (line 5), cluster retraining (line 6), finding the closest clusters (line 7), determining if merging these clusters is beneficial (line 8) and if so, cluster merging the two clusters (line 10). Below $\underline{\Omega}$ is the collection of all current individual clusters $\omega$

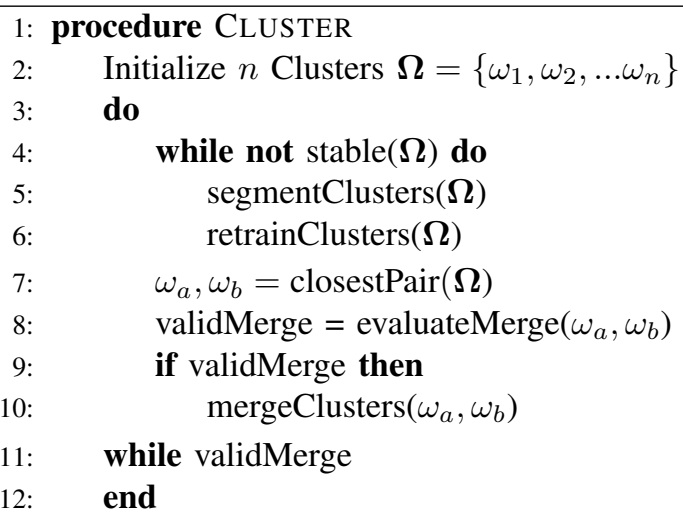

\section{Clustering Algorithms}

We analyse several potential clustering algorithms that can be utilized within our weed scouting pipeline. The following sections shall describe these algorithms and how they are implemented.

1) Diarization Clustering: The clustering methods used within diarization to group speakers together fall under two main categories, these being bottom-up or top-down diarization [17]. In this work we utilize the clustering technique used in bottom-up diarization which is a variation of agglomerative hierarchical clustering [27] where clusters are iteratively merged together based upon a relative distance metric until an ending criterion is reached.

In this work, we model the diarization clustering system heavily on the early diarization process described in [28]. This earlier process was used as, unlike in later methods [16], it did not utilize a hidden Markov model which requires a link between consecutive observations which is not possible in our application. We modify this early algorithm slightly however by, instead of using a single iteration of resegmentation and retraining, using multiple iterations until cluster stability is reached. Using this approach, clusters tend to be more stable before merging which leads to more accurate clusters being generated. Our modified diarization clustering algorithm used within this work is shown in Algorithm 1. In this bottom-up system, each cluster is modelled by a multivariate Gaussian. We also add a further improvement specific to our application which we call cluster locking which will be explained further in Section III-C.2.

We use symmetric Kullback-Leibler (KL2) distance [28] to measure the distance between clusters and the Bayesian information criterion [29] as our stopping criterion.

2) Cluster Locking: In our task, we aim to cluster each image of a plant into a group of similarly looking plants. We can, however, utilize the fact that our robotic platform 
will be able to take multiple images of the same plant to enhance our clustering accuracy. For instance, a robot may take several images of the same plant as it traverses over it, allowing for more information to be available for that one plant. Rather than treat each image as its own plant, we can initialize plant images which are in approximately the same real-world location as being the same plant. Using this we can generate initial clusters which have a better model representation of the single plant than each individual image would have.

In order to keep the high accuracy and improved plant models throughout clustering, we must also enforce that these images of the same plant, must be locked together throughout the optimization step of the diarization clustering procedure. We do this by adding in an extra step checking after segmentation if samples of the same plant have been kept together or if they have been separated. If the latter is true, then for each cluster containing the same plant sample points, we calculate the combined log-probability that each sample point belongs to that cluster. The cluster which contains the highest log-probability is then given all samples of that plant. This ensures that the samples of the plant are kept together while still allowing diarization to optimize the clusters after each iteration of cluster merging. This leads to improved cluster models that better represent each of the plants contained within. We refer to this new adaptation of diarization clustering as diarization-locked clustering throughout the remainder of this work.

3) Hierarchical Clustering: Agglomerative hierarchical clustering is a technique of progressively merging small clusters together based upon their relative distances to each other [9]. It merges the closest clusters iteratively to form a hierarchical clustering tree otherwise known as a dendrogram and chooses the number of clusters based on some predetermined stopping criterion. Typically this begins with treating each data point as a cluster but can be given an initial over-segmentation for initialization.

In order to be consistent, in our work we utilize the same initialization in tests as is done for diarization and utilize the same distance metric and stopping criterion. The only difference between the diarization clustering and hierarchical clustering used within this work is that diarization has the extra optimization step as explained in Section III-C.1.

We also improve hierarchical clustering through using the same initialization as is done for the diarization-locked clustering algorithm described in Section III-C.2 which we refer to as hierarchical-locked in our experiments. While there is no actual locking procedure required throughout the clustering algorithm due to the lack of optimization in hierarchical clustering, the improved initialization which takes advantage of multiple observations of the same plant improves clustering accuracy and so is considered its own algorithm in our tests.

4) Dirichlet Process Gaussian Mixture Model (DPGMM): In this work, one of the techniques we evaluate for use in the clustering step of the diarization pipeline is Dirichlet process Gaussian mixture model (DPGMM) clustering [30].
In our work this is defined as a form of infinite Gaussian mixture model (IGMM) [31], which is a form of Gaussian mixture model (GMM) which does not require the number of Gaussian components to be known in advance, keeping with the core principle of what a diarization system should be capable of.

This system assumes an infinite number of clusters exist but that only a few are present in the given dataset, where each cluster is modelled using a Gaussian distribution. The number of clusters chosen is dependant on an aggregation parameter $\alpha$. When $\alpha$ is large, the number of clusters chosen is typically also large and the same applies for small values of $\alpha$. Once the DPGMM is generated, the Gaussians within the model are used to formulate clusters with each data point being assigned to the cluster with the Gaussian which best represents them. Further details about DPGMMs and IGMMs can be found within [30] and [31]. For our experiments, $\alpha$ was chosen experimentally for each test.

5) Affinity Propagation ( $A P$ ): The final technique used in our clustering experiments is affinity propagation [14], [15]. As with the other techniques used here, affinity propagation does not require a known number of clusters. This technique generates exemplars by iteratively updating how likely each point can serve as an exemplar and how likely each point could belong to each point as an exemplar. Through this process, eventually a consensus occurs and clusters are assigned to all points. More details can be found within [14] and [15].

\section{Mapping}

To visualise the performance of the species clustering in the field a map was constructed from the image data set collected of the field. Each of the images in the data set were stitched together to form a large continuous mosaic of the field. The images from the data set are initially placed on to the mosaic using the position and rotation from the GPS and INS. To compensate for the inaccuacies in the GPS and INS values we match features extracted using [32] between each pair of adjacent images. Based on the median of the difference in poses of the features we apply an affine transformation to align the overlapping portions of the images. The output of the clustering was then overlayed on to the map to display both the spatial accuracy and the clustered plant species.

\section{EXPERIMENTAL SETUP}

In order to test our unsupervised scouting for agricultural robotics system and evaluate the effects of the locking described in Section III-C.2 we collected evaluation data using a robotics platform was required and developed a new method for evaluating the effectiveness of the clustering achieved which was appropriate for our application. The data collection and evaluation metric used in our experiment are explained in the following two subsections.

\section{A. Evaluation Data Collection}

Our tests for unsupervised weed scouting were performed using data collected from the Agbot II agricultural robotics 


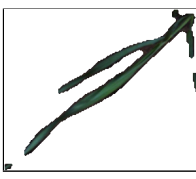

(a) Wild Oats

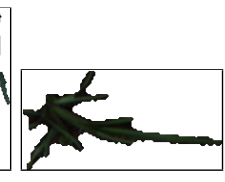

(b) Feathertop

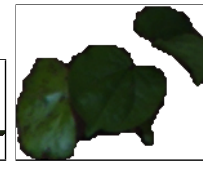

(c) Cotton

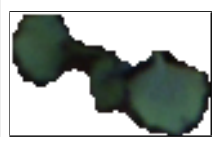

(d) Sowthistle
Fig. 3: Example of each plant species within our dataset

TABLE I: Summary of test dataset

\begin{tabular}{|c|c|c|c|c|}
\hline & Wild Oats & Feathertop & Cotton & Sowthistle \\
\hline \# Images & 113 & 90 & 157 & 11 \\
\hline \# Plants & 33 & 27 & 49 & 3 \\
\hline
\end{tabular}

platform. Data was collected from sections of a single field which was seeded with four specific weed species, two grasses and two broad-leaves. The species grown were wild oats, feathertop, cotton and sowthistle as shown in 3 A summary of the data collected can be seen in Table I] It should be noted that evaluation is done on the image level and the number of plants is given merely as reference. Plant-level observations are used only for diarization-locked and hierarchical-locked clustering methods as described in Section III-C.2

The process for dataset compilation was as follows. Image frames were extracted at a rate of $5 \mathrm{~Hz}$ as the AgBotII traversed the desired sections of the field. Plant regions were extracted from the image frames into cropped individual images with segmented backgrounds as shown in Fig. 3 using the segmentation algorithm described in Section III-A. These cropped images were manually labelled according to the four species described above.

The shape HCFs used in this work as described in Section III-B were extracted using the largest contour of the segmentation mask for each image and as our images only contained RGB information, the statistical features were calculated using the normalized excessive green vegetative index image as was done in [23]. As explained in Section III-B we also extract two forms of DCNN features. GoogLeNet features are extracted from the output of the average pooling layer of the fine-tuned GoogLeNet model. To generate bottleneck features we added a 128 neuron fully connected layer between the average pooling layer and the softmax layer of the GoogLeNet architecture before subjecting the network to the same fine-tuning as was done for the GoogLeNet model. Features were then extracted from this layer. We also generate a series of features where the hand-crafted and DCNN features are concatenated together. All features undergo $\mathrm{L}_{2}$ normalization before being fed into any of the clustering algorithms. For concatenated features, $\mathrm{L}_{2}$ normalization is performed on the separate feature types before merging and no further normalization is performed after this.

As the robot traversed the field, some plants were detected multiple times and each instance is included in the final dataset. In order to implement the improved initialization and image locking mentioned in Section III-C.2, cropped plant images were also manually grouped together if they were all from the same plant. Clusters therefore could be initialized with data from all images which are associated with a single plant. While this was done manually for our dataset generation, a similar result could be achieved for an online robotic application using relative GPS position of each extracted plant region such as is done on the BoniRob platform [33]. In all other instances, initialization treated each image as its own cluster, except for DPGMM which was initialized with 300 clusters.

\section{B. Evaluation Metric}

In order to quantifiably evaluate and compare the different clustering techniques examined within this work, an appropriate evaluation metric was required. Typically in clustering analysis with a set goal in mind such as ours, clustering analysis is performed using pairwise variations of classic evaluation measures such as pairwise precision, recall, accuracy, and F1 score which are described in more detail in [34].

While effective, these metrics do not prioritize the goals of this project. Our goal is to generate very few, pure clusters which can be examined by a farmer and then manually classified by them. This therefore requires a high priority on having very pure clusters which are separated into as few groups as possible so as to save the farmer time (i.e. 20 very pure clusters of the same species is not very desirable). Here we define the purity of cluster $k$ in terms of class $c$ as follows:

$$
\operatorname{Purity}(k \mid c)=\frac{n_{k}^{c}}{n_{k}}
$$

where $n_{k}^{c}$ is the number of samples held in cluster $k$ of class $c, n_{k}$ is the total number of samples within cluster $k$,

In order to better analyse our results in the terms which are important to our application, a new metric was generated which we shall refer to as the DScore. First, each cluster is assigned to the class which dominates the cluster. For example in Fig 4, the first cluster $\left(K_{1}\right)$ mostly contains black dots which are data points from class $1\left(C_{1}\right)$ and so $K_{1}$ has been assigned to $C_{1}$. In context this could mean that the $K_{1}$ has been assigned to the cotton class. We calculate an individual score for each class which is a weighted sum of cluster purities for all clusters divided by the number of clusters which the class has been separated into. This division penalizes oversegmentation while the weighted purity prioritizes the importance of as many points as possible being part of pure clusters. DScore is formulated as follows:

$$
\operatorname{Score}(c)=\frac{\sum_{k \epsilon G} \operatorname{Purity}(k \mid c) \times \frac{n_{k}^{c}}{n^{c}}}{N_{K}^{c}}
$$

where $G$ is the set of clusters which have been assigned to class $c, n^{c}$ is the total number of samples of class $c$, and $N_{K}^{c}$ is the number of clusters assigned to class $c$.

The final DScore is then calculated as the average of the scores across all classes. 

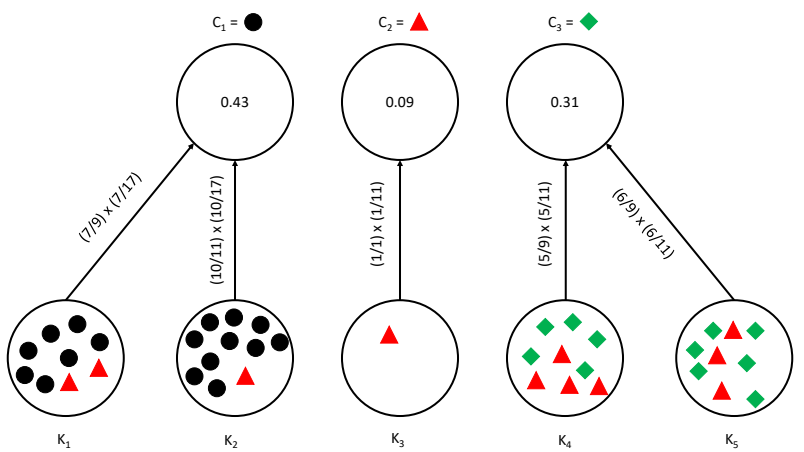

Fig. 4: Simplistic scenario to describe the calculation of the DScore. Each cluster is assigned to a class, as indicated by the arrows, based on which class is dominant within the cluster. The score for each class is the sum of its assigned cluster's purities, weighted by the proportion of the class' points owned by said cluster, divided by the number of clusters assigned to the class. For example, $K_{1}$ has a purity of $7 / 9$ as there are 7 samples of the dominant class $C_{1}$ (black dots) out of the total of 9 samples within cluster $K_{1}$. The weighted importance of $K_{1}$ towards the score for class $C_{1}$ is $7 / 17$ as it contains 7 of the total $17 C_{1}$ samples. Weighted addition of $K_{1}$ and $K_{2}$ s contributions is then divided by the number of clusters assigned to $C_{1}$ which is 2 to give Score $\left(C_{1}\right)=((7 / 9 \times 7 / 17)+(10 / 11 \times 10 / 17)) / 2=0.43$. The score for each class is shown in the class circle. The final DScore for the clustering above is 0.28

$$
\text { DScore }=\frac{\sum_{i=1}^{N_{C}} \operatorname{score}(i)}{N_{C}}
$$

where $N_{C}$ is the total number of classes. This averaging ensures that each class is considered equally important to the final score, avoiding issues with unbalanced data. If this was not done, the metric may presume very good clustering has been achieved despite one class being very poorly clustered such as the red triangles of $C_{2}$ in Fig 4

The DScore has a maximum value of 1 associated with perfect clustering and is a good measure for the effectiveness of clustering within the task of unsupervised weed scouting for agricultural robotics. A full example is shown in Fig 4 .

\section{RESULTS}

To demonstrate the effectiveness of our features and clustering techniques, we performed a thorough analysis across all feature combinations and clustering techniques described in Sections III-B and III-C respectively. This includes eight different combinations of features and six clustering algorithms including our diarization-locked and hierarchicallocked clustering algorithms. Analysis was performed both quantitatively and qualitatively as shall be demonstrated in the following subsections.

The segmentation algorithm, described in Section III-A. was derived using independent training and evalution data. On this independent data, it achieved an F1 score of 0.97 on
TABLE II: Best Clustering Result Comparison

\begin{tabular}{|c|c|}
\hline Clustering Algorithm & DScore \\
\hline AP & 0.16 \\
\hline DPGMM & 0.37 \\
\hline Diarization & 0.37 \\
\hline Diarization-locked & 0.40 \\
\hline Hierarchical & 0.41 \\
\hline Hierarchical-locked & $\mathbf{0 . 4 4}$ \\
\hline
\end{tabular}

the evaluation data which demonstrates the effectiveness of the approach.

\section{A. Clustering Quantitative Analysis}

All quantitative analysis of the various algorithms and features was achieved using our new evaluation metric known as the DScore, described in full in Section IV-B

When examining the best results of each clustering algorithm as shown in Table $\Pi$ it becomes clear that the best performing clustering algorithm was our hierarchical-locked algorithm. DPGMM and AP clustering were unable to exceed the hierarchical-based clustering algorithms, achieving DScores of 0.37 and 0.16 respectively. Diarization-locked and hierarchical-locked clustering exceeded the performance of their original algorithms, achieving DScores of 0.40 and 0.44 respectively in comparison to their original algorithm DScores of 0.37 and 0.41 respectively. This is reinforced when we look at the locking comparison graph of Fig. 6 In most cases, particularly when using bottleneck features merging with hcf and when using only hofs, we can see the benefit gained in using the locking algorithm for diarization and hierarchical clustering.

Also, it was shown that using bottleneck features provided equivalent or improved results for all diarization, hierarchical and DPGMM clustering experiments over that of GoogLeNet features as shown in Fig. 5 with DScore improvements of up to 0.34. Hand-crafted features, were never able to outperform the best DCNN features for each experiment achieving DScores of up to 0.21 and 0.22 for the original and scale-robust HCFs respectively. No great increase was achieved when concatenating DCNN features with handcrafted features and was, in fact, often detrimental to clustering accuracy. Overall, the highest DScores were achieved using our improved hierarchical-locked clustering algorithm in combination with DCNN bottleneck features.

\section{B. Clustering Qualitative Analysis}

As well as evaluating how well each method works in a quantitative manner, we also evaluated the final clustering map generated in a qualitative manner. Here we looked at the actual plant images in each cluster, particularly for our most successful hierarchical-locked clustering system. When we look at the three clusters generated by this technique as summarized in Fig. 7 we can see that cotton plants appear to have been grouped together in cluster B while the grasses have been distributed into clusters A and C. Our algorithm was found therefore to almost always group cotton plants together in the same group without any prior knowledge about the appearance of cotton plants. 


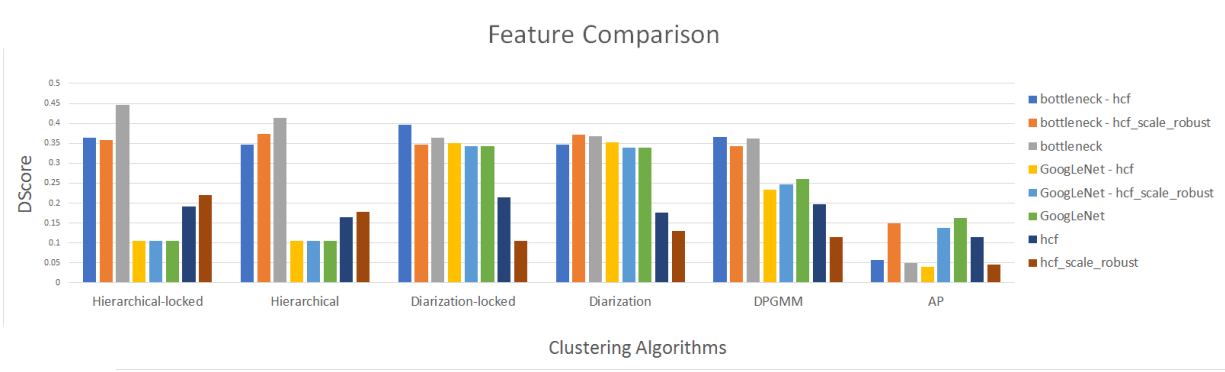

Fig. 5: DScore comparison of all the feature types examined. Best viewed in colour

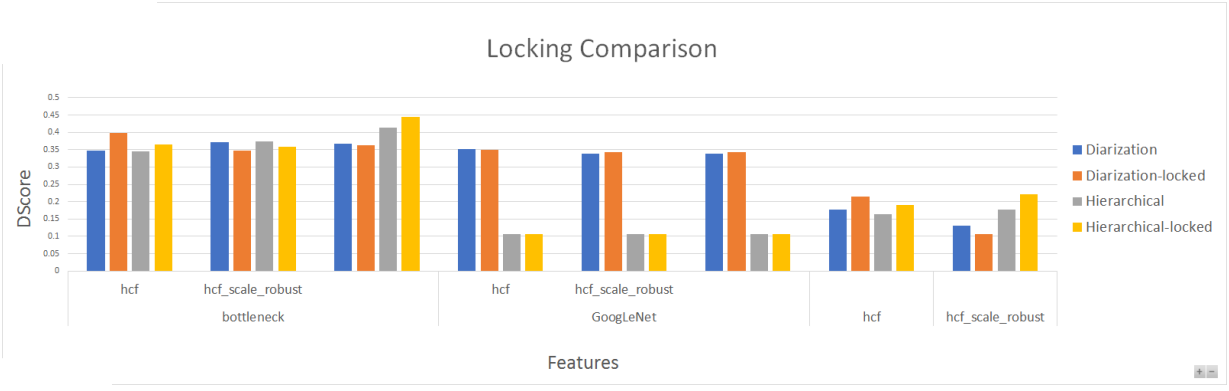

Fig. 6: DScore comparison of diarization and hierarchical clustering against their new locking algorithm versions.

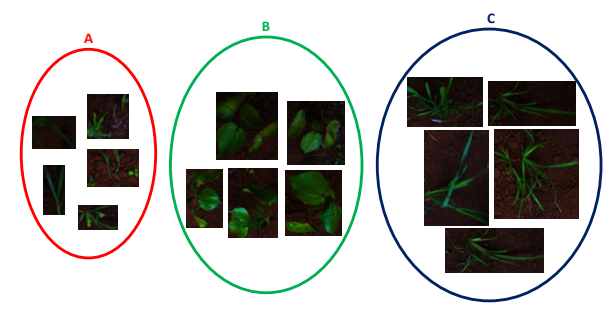

Fig. 7: Summary of clustering results for best clustering system (hierarchical-locked clustering using bottleneck features).

Through this example we can also see the main source of error in the clustering assignments. There is no clear distinction made between the two types of grass. This is to be expected as they are both very visually similar even to human eyes and they could be grouped together by the farmer if desirable when applied to real fields. As well as this, the sowthistle plants have been grouped together with the grasses as opposed to with the cotton or in its own cluster. We have attributed this to the fact that the sowthistle plants are particularly young here and have few distinguishing characteristics combined with the fact that there are very few samples of sowthistle, making it more difficult to build a distinctive plant model.

Finally in our qualitative analysis, we review the final map as it would be seen by a farmer shown in Fig. 8 . Here we only show a small region of the field, however an example of a fully mapped field is shown in the supplemental video supplied ${ }^{1}$. Here it is shown not only what is possible

\footnotetext{
http://tinyurl.com/WS-AGR-QUT
}

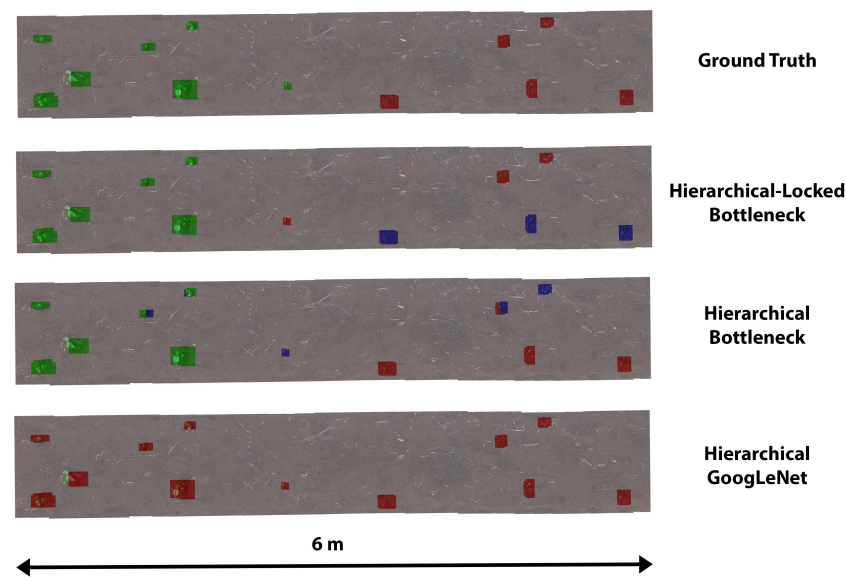

Fig. 8: Comparisons of small sections of the final map. Here, colours represent different groups for the clustering algorithms. The main cotton cluster for each has been kept as green. When two colours appear in the same region for Hierarchical with bottleneck features, it is representative of differing assignments being given to different images same plant. Best viewed in colour.

at present (fairly accurate cotton vs grass clustering) but also how much of an improvement can be gained through our hierarchical-locked method using bottleneck features over other techniques which sometimes give the same plant a different clustering assignment when the robot is given multiple images of the same plant. 


\section{CONCLUSIONS}

This paper demonstrates the first steps towards generating a completely unsupervised weed scouting system for use in agricultural robotics. Using data collected in the field from an agricultural robotics platform, we cluster plants together into groups using several state-of-the art clustering algorithms. We utilized low dimensional DCNN bottleneck features, which are proven to outperform both the high-dimensional DCNN features from the GoogLeNet framework, and handcrafted plant classification features for the task of clustering. We showed how clustering accuracy could further be improved for hierarchical-type clustering algorithms through locking images identified as the being the same individual plant together at initialization and through the clustering procedures themselves. We have also identified some of the key challenges to be overcome for future work to achieve results closer to species-specific clustering such as high interclass similarity and low numbers of species samples. Despite these challenges, we were able to successfully cluster the vast majority of cotton plants into a single, pure species group, separating it from them from any grasses present in the field without any prior training or knowledge about the field.

\section{ACKNOWLEDGMENTS}

We would like to acknowledge and thank the Grains Research and Development Corporation for contributing funds towards this research.

\section{REFERENCES}

[1] G. Charles and T. Leven, "Integrated weed management (iwm) for australian cotton," Cotton Pest Management Guide, pp. 88-119, 2011.

[2] N. Gilbert, "A hard look at GM crops," Nature, vol. 497, no. 7447, pp. 24-26, 2013

[3] GRDC. (2016) Integrated weed management hub. Accessed 07-092016. [Online]. Available: www.grdc.com.au/Resources/IWMhub

[4] S. M. Pedersen, S. Fountas, H. Have, and B. S. Blackmore, "Agricultural robotssystem analysis and economic feasibility," Precision Agriculture, vol. 7, no. 4, pp. 295-308, July 2006.

[5] J. P. Sansao, M. S. Jnior, L. A. Mozelli, F. A. Pinto, and D. M. Queiroz, "Weed Mapping Using Digital Images," in Proceedings of International Conference on Agricultural Engineering-CIGR, 2012.

[6] R. Gerhards and H. Oebel, "Practical experiences with a system for site-specific weed control in arable crops using real-time image analysis and gps-controlled patch spraying," Weed Research, vol. 46, no. 3, pp. 185-193, 2006.

[7] N. Grira, M. Crucianu, and N. Boujemaa, "Unsupervised and semisupervised clustering: a brief survey," A review of machine learning techniques for processing multimedia content, Report of the MUSCLE European Network of Excellence (FP6), 2004.

[8] J. MacQueen and others, "Some methods for classification and analysis of multivariate observations," in Proceedings of the fifth Berkeley symposium on mathematical statistics and probability, vol. 1. Oakland, CA, USA., 1967, pp. 281-297.

[9] S. C. Johnson, "Hierarchical clustering schemes," Psychometrika, vol. 32, no. 3, pp. 241-254, Sept. 1967.

[10] S. Salvador and P. Chan, "Determining the number of clusters/segments in hierarchical clustering/segmentation algorithms," in 16th IEEE International Conference on Tools with Artificial Intelligence, 2004. ICTAI 2004, Nov. 2004, pp. 576-584.

[11] H. Frigui and R. Krishnapuram, "A robust competitive clustering algorithm with applications in computer vision," Pattern Analysis and Machine Intelligence, IEEE Transactions on, vol. 21, no. 5, pp. 450465, 1999.

[12] H. Frigui and R. Krishnapuram, "Clustering by competitive agglomeration," Pattern recognition, vol. 30, no. 7, pp. 1109-1119, 1997.
[13] D. M. Steinberg, S. B. Williams, O. Pizarro, and M. V. Jakuba, "Towards autonomous habitat classification using Gaussian Mixture Models," in 2010 IEEE/RSJ International Conference on Intelligent Robots and Systems (IROS), Oct. 2010, pp. 4424-4431.

[14] B. J. Frey and D. Dueck, "Mixture modeling by affinity propagation," Advances in neural information processing systems, vol. 18, p. 379, 2006, 00087

[15] D. Dueck and B. J. Frey, "Non-metric affinity propagation for unsupervised image categorization," in 2007 IEEE 11th International Conference on Computer Vision. IEEE, 2007, pp. 1-8, 00165.

[16] C. Wooters and M. Huijbregts, "The ICSI RT07s Speaker Diarization System," in Multimodal Technologies for Perception of Humans, ser. Lecture Notes in Computer Science, R. Stiefelhagen, R. Bowers, and J. Fiscus, Eds. Springer Berlin Heidelberg, 2008, pp. 509-519.

[17] X. Anguera Miro, S. Bozonnet, N. Evans, C. Fredouille, G. Friedland, and O. Vinyals, "Speaker Diarization: A Review of Recent Research," IEEE Transactions on Audio, Speech, and Language Processing, vol. 20, no. 2, pp. 356-370, Feb. 2012.

[18] J. Donahue, Y. Jia, O. Vinyals, J. Hoffman, N. Zhang, E. Tzeng, and T. Darrell, "Decaf: A deep convolutional activation feature for generic visual recognition," arXiv preprint arXiv:1310.1531, 2013.

[19] A. Krizhevsky, I. Sutskever, and G. E. Hinton, "Imagenet classification with deep convolutional neural networks." in NIPS, vol. 1, no. 2, 2012, p. 4.

[20] C. Szegedy, W. Liu, Y. Jia, P. Sermanet, S. Reed, D. Anguelov, D. Erhan, V. Vanhoucke, and A. Rabinovich, "Going Deeper With Convolutions," in Proceedings of the IEEE Conference on Computer Vision and Pattern Recognition, 2015, pp. 1-9.

[21] I. Philipp and T. Rath, "Improving plant discrimination in image processing by use of different colour space transformations," Computers and electronics in agriculture, vol. 35, no. 1, pp. 1-15, 2002.

[22] S. Suzuki and K. Abe, "Topological structural analysis of digitized binary images by border following," in CVGIP, 1985, pp. 32-46.

[23] D. Hall, C. McCool, F. Dayoub, N. Sünderhauf, and B. Upcroft, "Evaluation of features for leaf classification in challenging conditions," in Winter Conference on the Applications of Computer Vision, 2015.

[24] S. Haug, A. Michaels, P. Biber, and J. Ostermann, "Plant classification system for crop/weed discrimination without segmentation," in IEEE Winter Conference on Applications of Computer Vision, 2014.

[25] P. Bonnet, W.-P. Vellinga, R. Planqu, A. Rauber, S. Palazzo, B. Fisher and H. Mller, "LifeCLEF 2015: Multimedia Life Species Identification Challenges," in Experimental IR Meets Multilinguality, Multimodality, and Interaction: 6th International Conference of the CLEF Association, CLEF'15, Toulouse, France, September 8-11, 2015, Proceedings, vol. 9283. Springer, 2015, p. 462.

[26] Z. Ge, C. Mccool, and P. Corke, "Content specific feature learning for fine-grained plant classification," in Working notes of CLEF 2015 conference, 2015

[27] S. Chen and P. Gopalakrishnan, "Speaker, environment and channel change detection and clustering via the bayesian information criterion," in Proc. DARPA Broadcast News Transcription and Understanding Workshop. Virginia, USA, 1998, p. 8.

[28] B. Zhou and J. Hansen, "Unsupervised Audio Stream Segmentation And Clustering Via The Bayesian Information Criterion," in in Proc. ISCLP 2000, 2000, pp. 714-717.

[29] G. Schwarz and others, "Estimating the dimension of a model," The annals of statistics, vol. 6, no. 2, pp. 461-464, 1978.

[30] T. S. Ferguson, "A Bayesian analysis of some nonparametric problems," The annals of statistics, pp. 209-230, 1973, 03840.

[31] C. E. Rasmussen, "The infinite Gaussian mixture model." in NIPS, vol. 12, 1999, pp. 554-560.

[32] E. Rublee, V. Rabaud, K. Konolige, and G. Bradski, "ORB: An efficient alternative to SIFT or SURF," in 2011 International Conference on Computer Vision, Nov. 2011, pp. 2564-2571.

[33] A. Ruckelshausen, P. Biber, M. Dorna, H. Gremmes, R. Klose, A. Linz, F. Rahe, R. Resch, M. Thiel, D. Trautz, and others, "BoniRoban autonomous field robot platform for individual plant phenotyping," Precision agriculture, vol. 9, no. 841, p. 1, 2009.

[34] C. D. Manning, P. Raghavan, and others, Introduction to information retrieval, 2008, vol. 1 . 